\title{
Idiopathic granulomatous mastitis - a new approach in diagnostics and treatment
}

\author{
L. VANOVCANOVA ${ }^{1,2}$, V. LEHOTSKA ${ }^{1,2, *}$, K. MACHALEKOVA ${ }^{3}$, I. WACZULIKOVA ${ }^{4}$, E. MINARIKOVA ${ }^{5}$, K. RAUOVA ${ }^{1,2}$, K. KAJO $^{3,6}$ \\ ${ }^{1}$ 2nd Radiology Department, Faculty of Medicine, Comenius University in Bratislava, Bratislava, Slovakia; ${ }^{2}$ St. Elizabeth Cancer Institute, \\ Bratislava, Slovakia; ${ }^{3}$ Department of Pathology, St. Elizabeth Cancer Institute, Bratislava, Slovakia; ${ }^{4}$ Department of Nuclear Physics and Bio- \\ physics, Faculty of Mathematics, Physics and Informatics, Comenius University in Bratislava, Bratislava, Slovakia; ${ }^{5}$ Department of Breast Dis- \\ eases, St. Elizabeth Cancer Institute, Bratislava, Slovakia; ${ }^{6}$ Biomedical Research Center, Slovak Academy of Sciences, Bratislava, Slovakia
}

*Correspondence: viera.lehotska@ousa.sk

Received February 1, 2019 / Accepted February 21, 2019

\begin{abstract}
Idiopathic granulomatous mastitis (IGM) is a rare inflammatory breast disease mimicking carcinoma and puerperal or non-puerperal mastitis. The primary purpose of this prospectively performed case control study was to compare clinical and imaging signs of IGM with the reference group of nonspecific, non- puerperal mastitis (NM) to identify the most typical clinical and imaging signs essential for a correct differential diagnosis. The secondary purpose was to present a new approach to non-invasive treatment. Thirty-nine women with histologically proven IGM and twenty-six patients with nonspecific mastitis underwent clinical examination, breast ultrasound (US), mammography (MG) and MRI examination. The most typical signs were selected for each group and method; followed by a statistical evaluation. The effectivity of colchicine, vitamin $\mathrm{E}$ and ribwort plantain tincture in treatment was assessed by clinical examination and imaging. Typical clinical signs of IGM included unilateral acute onset of breast edema, redness, palpable masses, missing fever, lymphadenopathy, no response to antibiotics or surgical interventions. Ultrasound revealed: "finger-like" structures (100\%), ductectasias (76.9\%), abscesses (76.9\%) and lymphadenopathy (15.4\%), while in MRI skin and tissue edema (100\%), multicentric lesions (100\%), abscesses (76.9\%), ring enhancement (84.6\%), lymphadenopathy (15.4\%) and small enhancing lymph nodes (38.5\%) were observed. Among the clinical signs, fistulas, hypoechoic mass, ductectasias and diffusion weighted images (DWI) restriction were significantly more frequent in patients with IGM than in those with NM. Treatment effectivity yielded $100 \%$ with a complete response between 6-19 months, depending on the disease extent. Targeted questions together with imaging can speed up selection for proper treatment with colchicine, vitamin E and local treatment. Long lasting use of antibiotics and repeated surgical interventions should be avoided.
\end{abstract}

Key words: idiopathic granulomatous mastitis, nonspecific mastitis, breast ultrasound, breast MRI, biopsy, conservative treatment

Idiopathic granulomatous mastitis (IGM) is a rare inflammatory disease of the breast characterized as a special type of mastitis which clinically mimics carcinoma and differs from the other types of mastitis previously described [1]. Subsequently, the term granulomatous lobular mastitis (GLM) was recommended to separate the lesion from granulomatous forms of periductal mastitis [2]. Currently the diagnosis is made by exclusion and should be proved by percutaneous biopsy procedures.

The primary pathologic change in IGM/GLM is a granulomatous inflammatory reaction centered on lobules, a granulomatous lobulitis. Granulomas composed of epithelioid histiocytes, Langhans giant cells accompanied by lymphocytes, plasma cells and occasional eosinophils are found within and around lobules. With progression of the inflammatory process, confluent granulomas may obscure or obliterate the lobulocentric distribution of the process, particularly toward the central portion of the tumor. Fat necrosis, abscess formation and fibrosis contribute to an effacement of the lobular distribution in confluent lesions. Stains and cultures for bacteria, acid-fast organisms and fungi are typically negative. Ducts incorporated in the lesion may become dilated and exhibit periductal and intraductal inflammation, but this is usually relatively inconspicuous [3].

This condition occurs primarily among young premenopausal women, most often in postpartum or breastfeeding 
mothers $[1,4]$, therefore the term postlactational granulomatous mastitis was also recommended [5], but the lesion may develop as long as 15 years postpartum [2]. According to some authors due to its positive response to steroid treatment, there might be a connection to an autoimmune disease [6]. Others proposed connections with Corynebacterium species, although by definition, IGM necessitates exclusion of any bacterias from histology specimens [7]. According to the concept of mammary duct-associated inflammatory disease sequence, certain conditions, such as pregnancy, breastfeeding and drug-induced hyperprolactinemia or galactorrhea, might be associated with an increased risk of IGM [8].

Other etiologic factors were proposed: hormonal treatment with oral contraceptives, smoking, hyperprolactinemia, however none of them were proved to cause IGM. Ethnicity seemed to be connected with higher incidence of IGM as most of the published studies came from Mediterranean region and the developing countries in Asia but even here more studies are required [9].

Diagnosis of IGM is frequently delayed because clinical symptoms vary and usually differ from case to case. Due to other diseases mimicking imaging signs IGM, the diagnostics becomes a challenge. Additionally, after correct diagnosis a proper treatment may represent a problem: common approaches use long lasting antibiotic and corticosteroid therapy as well as repeated surgical interventions. According to published sources, any combination of these drugs seems to be long term inefficient and connected with severe side effects. In this article we want to introduce both diagnostic as well as the new therapeutic approaches based on colchicine, vitamin $\mathrm{E}$ and local treatment with ribwort plantain (Plantago lanceolate) tincture.

\section{Patients and methods}

Patients characteristics. In a period from 02/2012 to $09 / 2018,39$ cases of IGM were collected, all based on histopathological examination of core cut biopsy samples. The reference group consisted of 26 patients having been diagnosed with histologically proven nonspecific mastitis. Clinical records, imaging and pathology specimens were reviewed by subspecialists in clinical breast health, breast radiology and breast pathology.

Image acquisition. Specific targeted clinical questions and selected imaging signs in ultrasound (IU22, Philips, linear transducer 12,5 and $17 \mathrm{MHz}$ ), mammography (Hologic Dimensions, bilateral mammography in craniocaudal and mediolateral oblique views) and breast MRI (Siemens Verio 3T) were analyzed for both groups. MRI examination was performed as follows: T2 TSE FS axial, TIRM coronal, T1 TSE hr axial, ep2d diff tra spair (b 0, 50, 800), ep2d diff tra spair ADC, T1 fl3d axial dynaVIEWS spair $1+5$ with i. v. administration of gadoteridol $(0,1 \mathrm{mmol} / \mathrm{kg})$, followed by 20 $\mathrm{ml}$ of saline. The statistical analysis was performed with the aim to identify the most relevant signs for IGM.
Treatment. After confirming diagnosis of IGM, colchicine (colchicum-dispert) 3x50 mg per day, vitamin E $400 \mathrm{mg}$ per day, local treatment with ribwort plantain tincture (ribwort plantain leaves extracted in $40 \%$ ethanol, in a ratio of $1: 5$ ) was applied 3-times a day as a poultice. The efficiency of treatment was followed by clinical, US and MRI examinations.

Immunohistochemical analysis. All tissue samples underwent immunohistochemical analysis that confirmed diagnosis of IGM and in all cases, bacterial, fungal and acidresistant microorganisms were excluded by special methods (Gramm, PAS, Ziehl-Nielsen).

Statistical analysis. Descriptive and bivariable analyses were performed on all selected patients' characteristics. Mean \pm standard deviation (SD) and median with interquartile ranges is given for age. Due to the skewness of data distribution the groups were tested using a non-parametric Mann-Whitney U test. Categorical variables are presented as counts and percentages, grouped in two-way contingency tables and analyzed using exact tests. In case of numerical calculability, chi-square tests were applied. Odds Ratios (OR) as the odds of presenting a sign in cases (IGM patients) to the odds of presenting a sign in controls (NM patients) were also calculated. All reported probability values were two-sided, and significance was set at $\mathrm{p}<0.05$. Statistical analyses were performed using StatsDirect 3.1.20 software (Stats Direct Ltd., Cheshire, UK).

\section{Results}

Patients' characteristics and radiological findings are summarized in Table 1. The median age of patients with IGM diagnosis was significantly lower than in the reference NM group ( 37 vs. 43.5 years; $\mathrm{p}=0.002$ ). All cases were premenopausal, all with prior full-term pregnancies, 12 of 39 (30.8\%) with the pregnancy within a 3 year-period before the onset of IGM.

Histomorphological diagnosis of IGM was based on the lobulo-centric inflammatory process with the formation of granulomas consisting of histiocytes, giant multinuclear cells, lymphoid elements and eosinophils. In Figure 1A non-necrotizing granulomatous inflammation composed of lymphocytes, histiocytes and giant cells is proven and as additional findings the microabscesses with neutrophils are detected (Figure 1B). Giant multinuclear cells had shown Langhans cell characteristics and together with mononuclear histiocytes, expressed immunohistochemical positivity of CD68. The inflammatory infiltrate was composed of lymphoid cells with immunohistochemical expression of CD45, CD20 and CD3. In all cases, bacterial, fungal and acid-resistant microorganisms were excluded.

Clinical presentation in IGM group was uniform, all cases had unilateral symptomatology unlike control patients having had bilateral symptomatology in $7.7 \%(\mathrm{p}=0.195)$. Further, 24 cases (61.5\%) presented with left breast enlarging mass. Breast erythema and skin edema were differentiated in 
Table 1. Clinicopathological characteristics of patients with idiopathic granulomatous mastitis and non-specific mastitis.

\begin{tabular}{|c|c|c|c|c|c|}
\hline \multicolumn{2}{|c|}{ Characteristic } & \multirow{2}{*}{$\frac{\text { IGM }(\mathbf{n}=\mathbf{3 9})}{37(36-40)}$} & \multirow{2}{*}{$\frac{\text { NM }(\mathbf{n}=\mathbf{2 6})}{43.5(38-58)}$} & \multirow{2}{*}{$\frac{\mathbf{O R}^{\star}}{\text { n.a. }}$} & \multirow{2}{*}{$\frac{\text { p-value }}{0.002}$} \\
\hline Age (years), median & & & & & \\
\hline Lactation & yes & $12(30.8 \%)$ & $6(24.0 \%)$ & \multirow{3}{*}{1.41} & \multirow{3}{*}{0.776} \\
\hline \multirow{5}{*}{ Location } & no & $27(69.2 \%)$ & $19(76.0 \%)$ & & \\
\hline & missing & 0 & 1 & & \\
\hline & right & $15(38.5 \%)$ & $11(42.3 \%)$ & \multirow{3}{*}{ n.a. } & \multirow{3}{*}{$0.195^{* *}$} \\
\hline & left & $24(61.5 \%)$ & $13(50.0 \%)$ & & \\
\hline & bilateral & $0(0 \%)$ & $2(7.7 \%)$ & & \\
\hline \multirow[t]{2}{*}{ Exudation } & yes & $9(23.1 \%)$ & $9(34.6 \%)$ & \multirow{2}{*}{0.57} & \multirow{2}{*}{0.399} \\
\hline & no & $30(76.9 \%)$ & $17(65.4 \%)$ & & \\
\hline \multirow[t]{2}{*}{ Fistulas } & yes & $15(38.5 \%)$ & $3(11.5 \%)$ & \multirow{2}{*}{4.79} & \multirow{2}{*}{0.024} \\
\hline & no & $24(61.5 \%)$ & $23(88.5 \%)$ & & \\
\hline \multirow[t]{2}{*}{ Surgery } & yes & $18(46.2 \%)$ & $7(26.9 \%)$ & \multirow{2}{*}{2.3} & \multirow{2}{*}{0.193} \\
\hline & no & $21(53.8 \%)$ & $19(73.1 \%)$ & & \\
\hline \multicolumn{6}{|l|}{ Ultrasound signs } \\
\hline \multirow[t]{3}{*}{ Hypoechoic mass } & yes & $39(100.0 \%)$ & $12(48.0 \%)$ & \multirow{3}{*}{$\infty$} & \multirow{3}{*}{$<<0.001$} \\
\hline & no & $0(0 \%)$ & $13(52.0 \%)$ & & \\
\hline & missing & 0 & 1 & & \\
\hline \multirow[t]{3}{*}{ Abscess } & yes & $30(76.9 \%)$ & $20(80.0 \%)$ & \multirow{3}{*}{0.83} & \multirow{3}{*}{0.999} \\
\hline & no & $9(23.1 \%)$ & $5(20.0 \%)$ & & \\
\hline & missing & 0 & 1 & & \\
\hline \multirow[t]{3}{*}{ Duct ectasia } & yes & $30(76.9 \%)$ & $7(28.0 \%)$ & \multirow{3}{*}{8.57} & \multirow{3}{*}{$<0.001$} \\
\hline & no & $9(23.1 \%)$ & $18(72.0 \%)$ & & \\
\hline & missing & 0 & 1 & & \\
\hline \multirow[t]{2}{*}{ Lymphadenopathy } & yes & $6(15.4 \%)$ & $1(3.9 \%)$ & & \\
\hline & no & $33(84.6 \%)$ & $25(96.1 \%)$ & 4.55 & 0.228 \\
\hline MRI signs & & & & & \\
\hline Ring enhancement & yes & $33(84.6 \%)$ & $19(82.6 \%)$ & & \\
\hline & no & $6(13.4 \%)$ & $4(17.4 \%)$ & 1.16 & 0.999 \\
\hline & missing & 0 & 3 & & \\
\hline DWI restriction & yes & $33(84.6 \%)$ & $13(56.5 \%)$ & & \\
\hline & no & $6(13.4 \%)$ & $10(43.5 \%)$ & 4.23 & 0.019 \\
\hline & missing & 0 & 3 & & \\
\hline
\end{tabular}

Continuous data are presented as means \pm standard deviations. Categorical data are presented as absolute counts with percent of column total. ${ }^{*}-$ the ratio of the odds of having the outcome in the upper row category of a characteristic in cases (IGM) to the odds of having that outcome in controls (NM). Patients with missing values were not included in the analysis. ** - Fisher-Freeman-Halton exact test for $2 \times 3$ contingency table. $\infty$ (infinity) - amount of OR cannot be estimated due to a zero proportion category. Abbreviations: IGM, idiopathic granulomatous mastitis; NM, non-specific mastitis; OR, Odds Ratio; p, probability; SD., standard deviation; n.a., not applicable; MRI, magnetic resonance imaging; and DWI, diffusion weighted images.
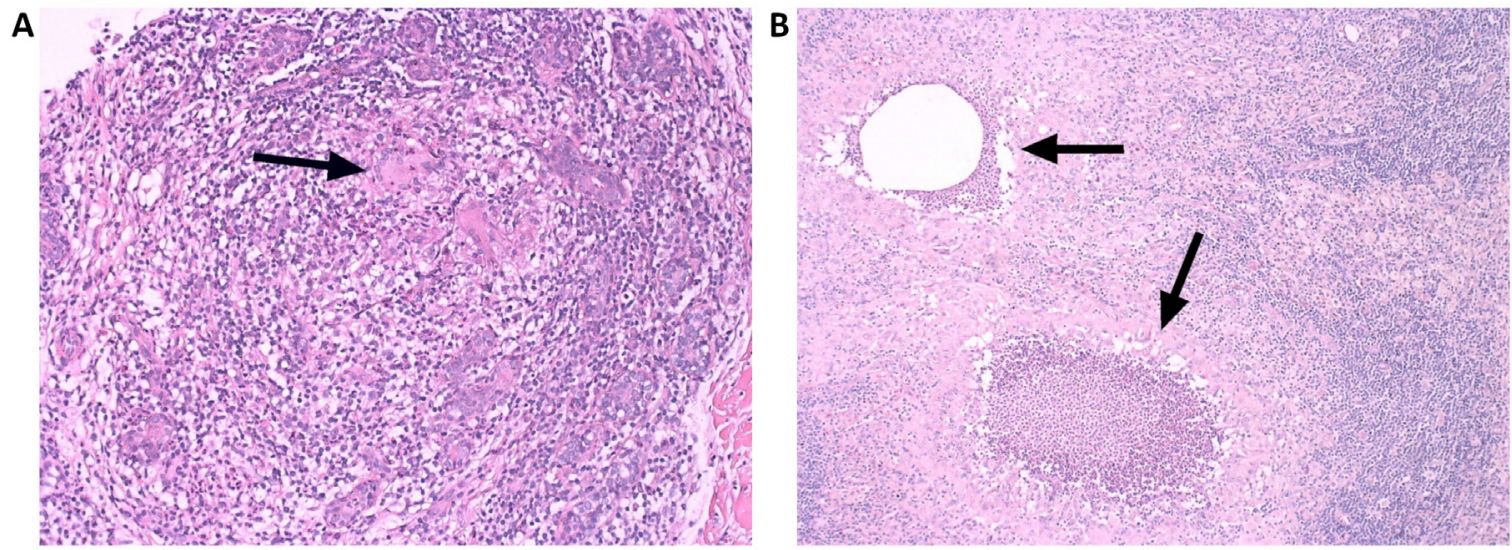

Figure 1. A) non-necrotizing granulomatous inflammation composed of lymphocytes, histiocytes and giant cells (arrow). H\&E stain, 400×, B) microabscesses formation (arrows) in some granulomas. H\&E stain; magnification 200×. 
30 cases $(76.9 \%)$. Fistulas appeared in 15 patients $(38.5 \%)$, which was significantly higher proportion than that found in the NM group ( $11.5 \%$ patients; $\mathrm{p}=0.024)$.

Nine cases $(23.1 \%)$ reported similar problem in the past while 30 cases $(76.9 \%)$ had the first attack of disease. Thirty cases $(76.9 \%)$ were treated by multiple antibiotics, 27 (69.2\%) reported having been prescribed more than 3 different antibiotics. The average time until the cases had been correctly diagnosed was 8.69 months (3-40 months).

All 39 cases have presented at the US examinations with hypoechoic masses with irregular and blurred borders, inhomogeneous character and a different degree of hypoechogenicity. On the other hand, in the reference NM group of hypoechoic masses appeared in 12 patients only (48.0\%), $\mathrm{p}<0.001$. Mean size of the affected area in IGM was $45.25 \mathrm{~mm}$. Thirty cases (76.9\%) have formed thick wall formations with hypo or anechoic content (abscesses), the same number of patients had multiple irregular retroareolar ductectasias, filled with echoic content, while in the NM group ductectasias were present only in 7 patients $(280 \%)$. Together with the degree of hypoechogenicity this characteristic was statistically highly significant $(p<0.001)$, which means that odds of having these signs among the cases were higher than odds of having these signs among the "controls" as expressed by OR values above 1 .

The most relevant ultrasound signs are displayed in Figure 2 - skin thickening with subcutaneous edema and hypoechoic large non-homogeneous areas, irregular lesion with non-homogeneous content (abscess) with fistula penetrating the skin and typical "finger-like" tubular formations emerging from hypoechoic areas.

In 36 IGM patients (92.3\%) at least one centrally necrotic lesion was described. In 24 (66.7\%) cases out of these patients fine needle aspiration biopsy (FNAB) was performed to get a sample for cultivation. No pathological infectious agent has been proven. In the IGM group ipsilateral axillary lymphadenopathy with lymph node of short axis diameter more than $10 \mathrm{~mm}$ appeared in 6 patients (15.4\%). Thirty-three (84.6\%)
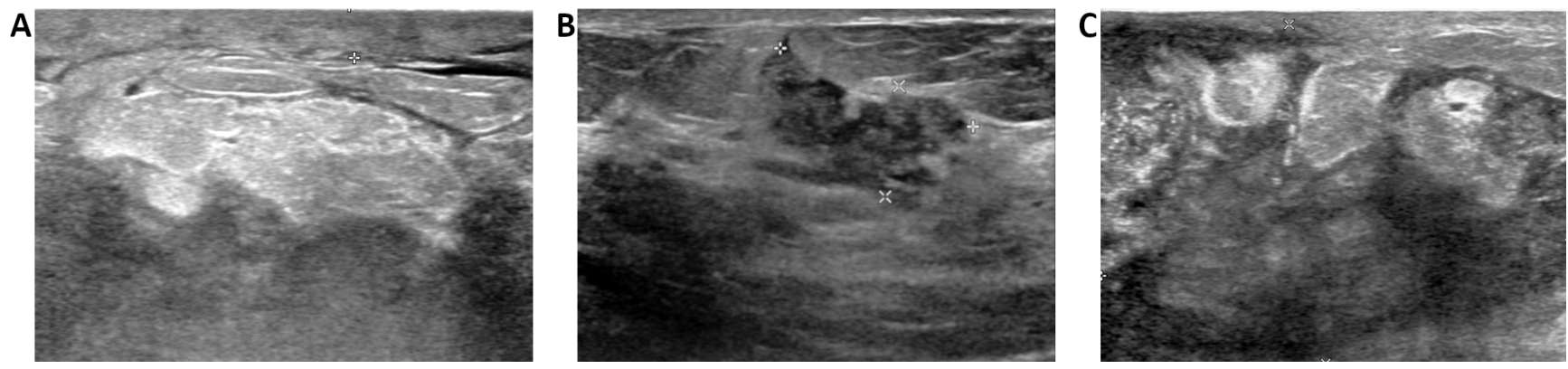

Figure 2. Breast ultrasound examination. A) skin thickening with subcutaneous edema and hypoechoic large non-homogeneous areas, B) irregular lesion with non-homogeneous content (abscess) with fistula penetrating the skin, C) typical "finger-like" tubular formations emerging from hypoechoic areas.
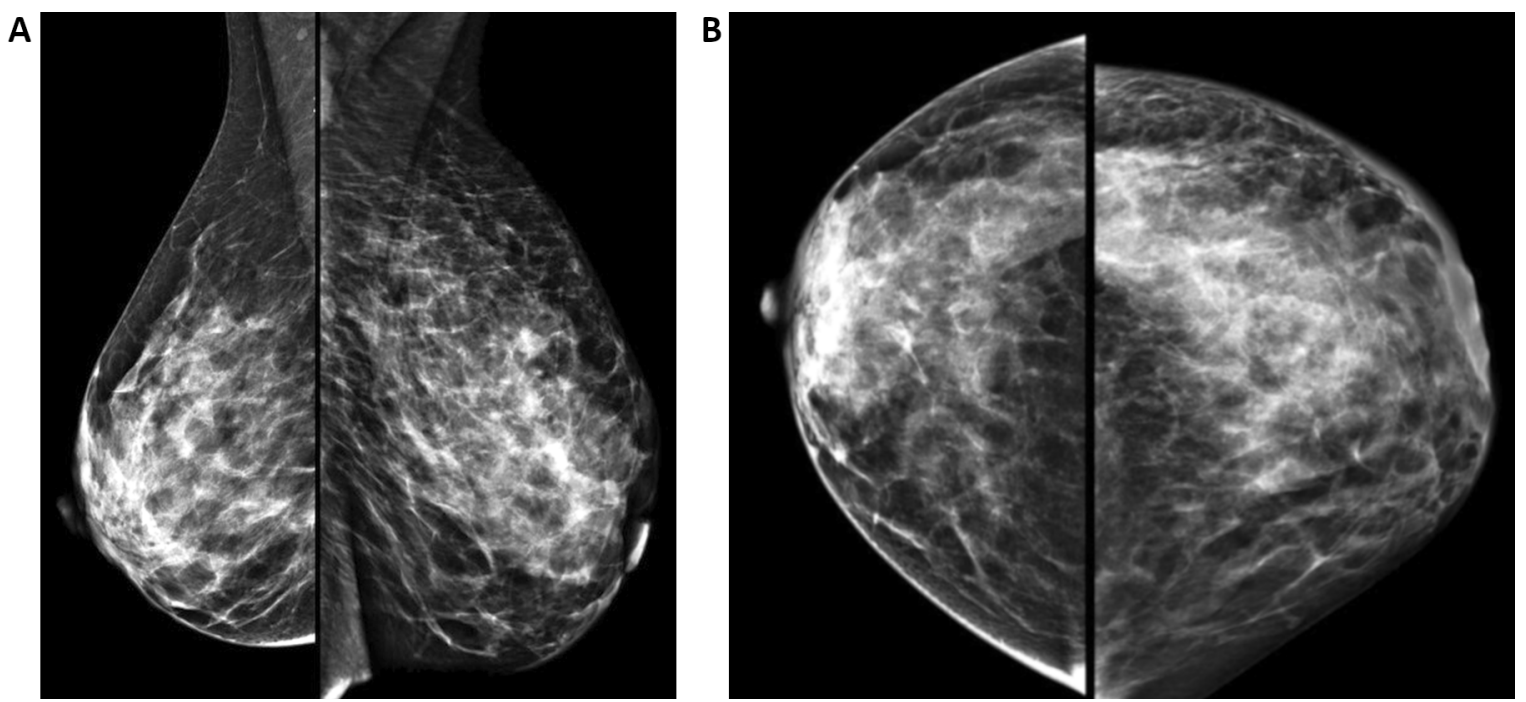

Figure 3. Digital mammography in mediolateral oblique (A) and craniocaudal view (B): non-specific skin thickening and increased density of the whole left breast as a sign of breast edema. 
patients had only multiple smaller lymph nodes with thicker cortex but preserved hyperechoic hilum. In the reference group only 1 patient (3.9\%) had ipsilateral lymphadenopathy.

Bilateral digital mammography was performed in 27 (69.2\%) IGM patients. Edema of the breast was presented as a blurred architecture and increased density of gland in 21 out of 27 cases (77.8\%), retraction of areolo-mammilar complex in 18 out of 27 cases $(66.7 \%)$. Similar number of patients presented with diffuse mammographic changes $(18,66.7 \%)$ and $9(33.3 \%)$ had focal abnormalities. Figure 3 shows nonspecific mammographic changes.

In all IGM cases an enlargement of the affected breast with diffuse skin edema was the most common MRI sign. All patients had multiple lesions in more than one quadrant of the breast and total size of the affected area was in all cases more extensive than it was supposed from ultrasound: the average size was $65.45 \mathrm{~mm}(25-105 \mathrm{~mm})$.

In diffusion weighted images (DWI) restriction of diffusion appeared in $33(84.6 \%)$ cases in at least one lesion in the breast, 6 patients $(15.4 \%)$ had no lesion with restriction of diffusion. The ring enhancement appeared in 33 (84.6\%) patients. In the control group ring enhancement was present in a similar proportion in 19 cases $(82.6 \%)$. However, restriction of diffusion was observed in statistically lower proportion in $13(50.0 \%)$ patients $(\mathrm{p}=0.019)$. These characteristics are displayed in Figure 4. Consequently, IGM patients had crude odds of having presented with this radiological sign 4.23 times that of NM patients (Table 1). Kinetic curve type 3 with wash out phenomenon appeared in 20 patients $(51.3 \%)$, type 2 with plateau in $16(41.0 \%)$ and in 3 cases $(7.7 \%)$ the lesions exhibited type 1 with progressive enhancement. Twenty out of the 39 patients $(51.3 \%)$ have pathological or suspicious lymph nodes described in MRI. Other clinical and imaging signs of nonspecific mastitis were statistically non-significant and are summarized in Table 1.

First effect of treatment appeared in all patients within one week (median 5 days): reduction of pain, swelling and erythema, followed by spontaneous evacuation of purulent content, or closing of existing fistulas. The length of the treatment was very variable, 6-24 months (mean 16 month) and lasted until the complete regression of changes was proved by MRI (Figures 5). Treatment effectivity yielded 100\% with a complete response between 6-19 months, depending on the disease extent. During analyzed period no case of disease recurrence appeared.

\section{Discussion}

IGM as a rare disease is regarded to be a diagnostic challenge and the correct diagnosis is finally reached "per exclusionem". In addition to carcinoma and inflammatory carcinoma, IGM should be differentiated from other inflammatory breast diseases such as nonspecific mastitis and rare chronical inflammatory diseases (Wegener granuloma, sarcoidosis, tuberculosis) [10]. Imaging characteristics of
IGM and NM are according to published papers often similar in all used methods (US, MG, MRI) and a histopathological evaluation is necessary to establish the diagnosis before deciding upon treatment [11]. From our experiences IGM can be suggested as a part of differential diagnosis at the first contact with a patient. Proper questions about the history of the problems can lead towards correct diagnosis and should be asked at the first examination. One should be interested in the onset of the problems, the first signs, presence or absence of fever, redness, edema of skin, nipple discharge, palpable masses and the history of pregnancy and breastfeeding. Other information about the applied treatment in the past, its effect, surgical interventions, spontaneous evacuation of purulent content and the cultivation results are very useful. From our observation we can highlight the most important and prominent clinical signs: the patient
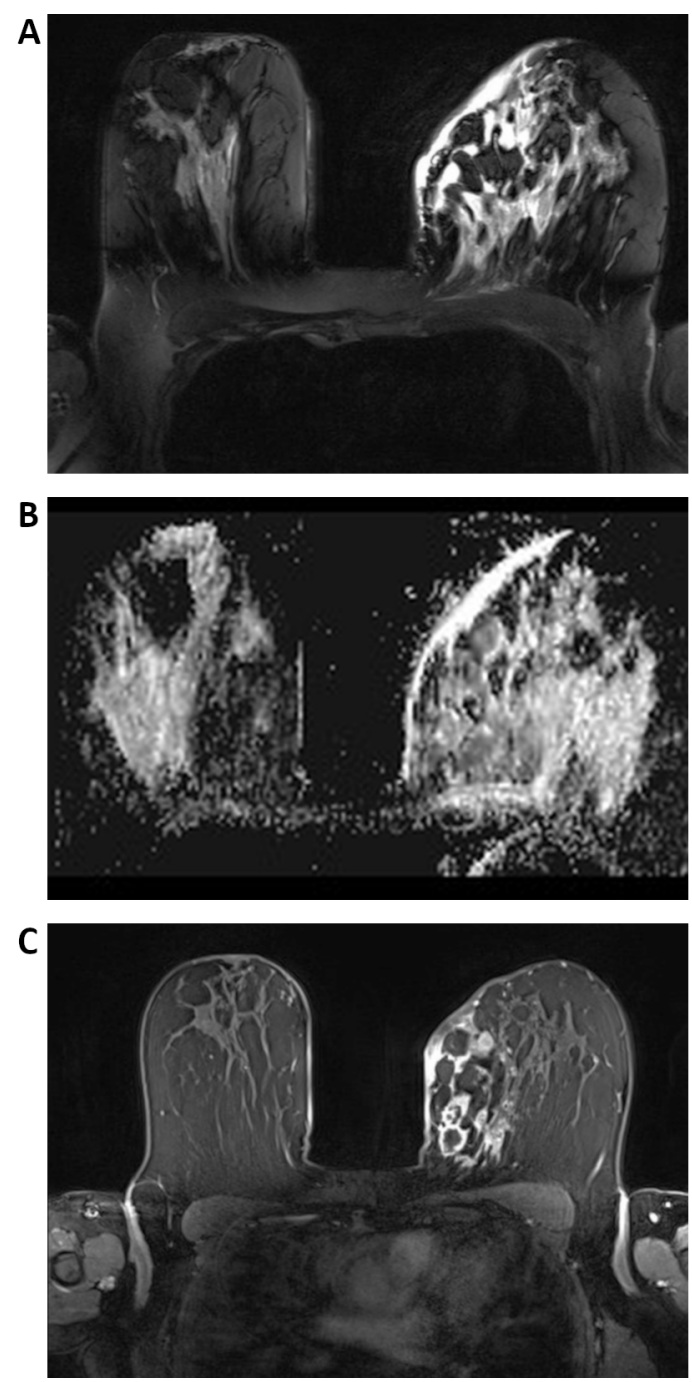

Figure 4. Contrast enhanced breast MRI. A) T2w reveals edema of skin, subcutaneous fat and fibroglandular tissue, B) ADC map shows restriction of diffusion within the lesions with strong rim enhancement (C). 

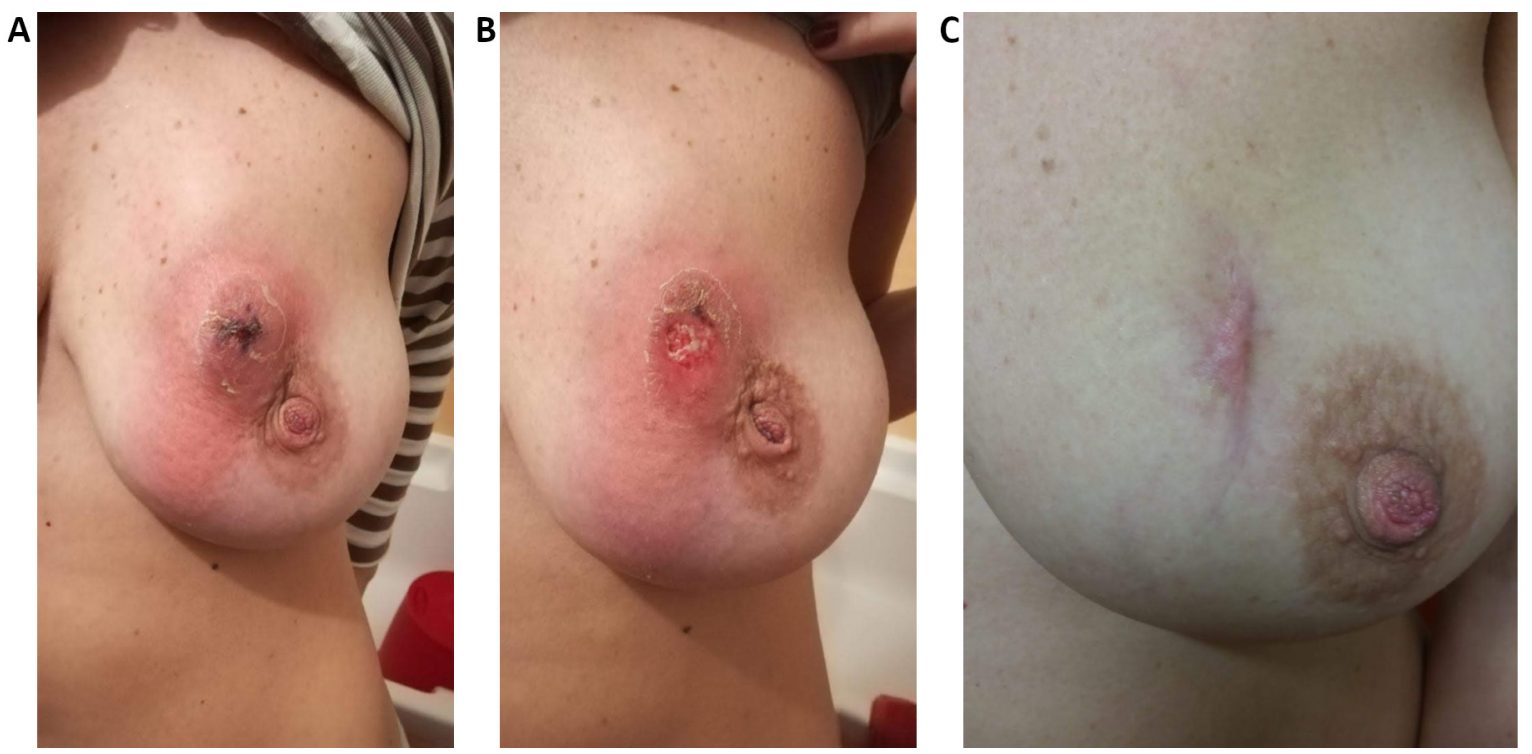

Figure 5. Clinical observation. A) 37y old patient, enlargement of right breast, edema with palpable mass and skin inflammation, B) after three weeks on treatment (colchicine $3 \times 50 \mathrm{mg} /$ day, vitamin E 1x400 mg/ day, local treatment with ribwort plantain), skin ulceration with evacuation of purulent content, C) after 6 months on treatment (colchicine $3 \times 50 \mathrm{mg} /$ day, vitamin E 1×400 mg/ day, local treatment with ribwort plantain).

is typically premenopausal with a sudden onset of one side breast enlargement, skin erythema and edema, with palpable masses. These problems are usually recurrent, and disease has a long history, without any or little improvement after a repeated antibiotic treatment, with relapses after surgical excisions [12]. Similar observations result from the most of recently published studies $[7,13,14]$.

After the proper history questionnaire breast ultrasound was a method of choice in the management of our patients since all of them were premenopausal.

These ultrasound signs are characteristic for IGM: diffuse skin thickening of the affected breast with retroareolar ductectasias partially or completely filled with hypoechoic content, hypoechoic irregular areas or multiple merging focal lesions together with tubular "fingerlike" structures, that can reliably differentiate from nonspecific mastitis. The role of FNAB at the diagnostics is limited and for the diagnosis insufficient due to its often confusing results [14], but in our cases it was used for cultivation purposes because sterility of material is one of the criteria for IGM [15]. The extent of the affected area in US was difficult to determine due to irregularity and merging of lesions. Ultrasound undervalued its extent: the average size was approximately $2 \mathrm{~cm}$ smaller than MRI described. Thus, MRI plays an important role of real extent determination because some lesions are not visualized by ultrasound adequately [14]. Axillary lymphadenopathy it is not a common sign of IGM. Lymph nodes detected by US were multiplied, with thickened cortex but small, with preserved morphology, its short axis diameter was less than $10 \mathrm{~mm}$. Only a minor part of patients developed pathological axillary lymphadenopathy which is in accordance with other studies $[4,13]$.
Mammography (MG) should be used only in cases where breast carcinoma is suggested while it brings usually no specific information and it is complicated by the dense terrain typical for young women [16]. The mammographic IGM signs as skin thickening, nipple retraction, trabecular structure of entire breast or focal densities and asymmetry can be signs of breast carcinoma or inflammatory breast carcinoma as well as of puerperal or non-puerperal mastitis. MG is important to exclude microcalcifications in cases suspicious for malignancy - microcalcifications are not typical for IGM [16].

Role of MRI is in the detection of additional changes that cannot be visualized by US and MG due to parenchymal edema. In addition, since IGM generally affects younger patients with dense breast, MRI may be useful in cases where MG or ultrasound are inconclusive. According to our observations breast MRI gives the correct disease extent information, can reveal small and discrete changes such as fistulas in the affected breast but also unrecognized disease in the contralateral breast. Its role in differentiation between IGM and malignancy is ambiguous. In case of IGM the infiltration and retraction of breast tissue are less prominent, and the edema is a dominant sign.

The pattern of enhancement is very variable, the rim enhancement was the dominant characteristic of the lesions in MRI, which is in accordance with other studies $[1,18]$. Generally, it is regarded as a sign suspicious for carcinoma. In our group of patients rim enhancement was tightly connected with a restriction of diffusion, which together with T2 hyperintensity and $\mathrm{T} 1$ hypointensity fulfill the criteria of abscess.

The kinetic curves have only minor additional value in IGM diagnostics. There are articles describing kinetic type 1 
as a typical for IGM $[4,18]$, others found type 3 as the most prominent sign of IGM lesions [19]. Time to intensity curve with washout phenomenon (TIC 3) was observed in most of the cases of our patients. Morphology of the lesion or infiltrate remains superior to its kinetic characteristics.

Axillar status was in agreement with sonographic findings - only six patients fulfilled criteria for pathological lymphadenopathy. However, MRI increased the number of patients with suspicious lymph nodes, due to their fast and early enhancement even though their shorter axis diameter was less than $10 \mathrm{~mm}$.

Finally, the role of MRI in treatment respond monitoring must be mentioned. With its ability to assess the extent at the disease it is the best and the most proper imaging method. The clinical improvement itself might seem to be sufficient for further management but according to our observation it is not rare that small residua of the disease remain unrecognized. The premature terminating of treatment can lead to early recurrence of the disease.

The treatment itself is based on the anti-inflammatory effect of colchicine: an alkaloid extracted from plants of the genus Colchicum (autumn crocus). Its therapeutic effect has been well documented in gout and familial Mediterranean fever [21]. Colchicine prevents microtubule assembly and thereby disrupts inflammatory activation, microtubulebased inflammatory cell chemotaxis, generation of leukotrienes and cytokines and phagocytosis. Its side effects are of gastrointestinal character as diarrhea, nausea, vomiting but at the suggested dose these are usually mild. No severe side effects leading to treatment interruption were observed in any of our patients. Liver and renal parameters were monitored regularly during the treatment.

Vitamin E was added in dose $400 \mathrm{mg}$ per day due to its antioxidant and skin protective effect. Together with local application of ribwort plantain tincture it fastens the healing process of skin ulcerations and fistulas and improves the cosmetic effect. The ribwort plantain effect appears to be related to an inhibition of prostaglandins synthesis because the plant extract reduced the inflammatory responses induced by either carrageenin or croton oil, as well as pain stimuli induced by chemical substances [22]. The onset of full effect of this complex treatment was very individual and depended on the extent of disease. It was followed basically by clinical examination and US, MRI was performed only in case of recurrence of severe symptoms or before termination of treatment.

In conclusion, according to the results of our prospectively performed case control study it must be underlined that IGM still remains histological diagnosis, but wide knowledge about its clinical as well as imaging presentation can lead to early suggestion of the correct diagnosis, and thus spare the patient from non-indicated surgical interventions as well as long lasting inefficient antibiotic treatment. A multidisciplinary cooperation of breast specialists can accelerate the whole diagnostic process and shorten the time of patients' stress and uncertainty from suspicious breast lesion. Our new therapeutic approach based on evidence of complete treatment response without any side effects or mutilations can improve patient's life quality in a short time. This treatment yields to complete resorption of IGM and can be repeatable used in case of disease relapse.

The rarity of IGM requires inter-institutional cooperation with the aim to collect more data for further multicentric study.

Acknowledgements: The author(s) received no financial support for the research, authorship, and/or publication of this article.

\section{References}

[1] KESSLER E, WOLLOCH Y. Granulomatous mastitis: A lesion clinically simulating carcinoma. Am J Clin Pathol 1972; 58: 642-646.

[2] GOING JJ, ANDERSON TJ, WILLKINSON S, CHETTY U. Granulomatous lobular mastitis. J Clin Pathol 1987; 40: 535-540. https://doi.org/10.1136/jcp.40.5.535

[3] HODA S. Inflammatory and Reactive Tumors, pp 37-78. In: SA Hoda, E. Brogi, FC Koerner, PP Rosen (Eds.). Rosen's Breast Pathology, Fourth Editon. Lippincott Williams \& Wilkins, Philadelphia, 2014, p 1400. ISBN 978-1-4511-76537

[4] FAZZIO RT, SHAH SS, SANDHU NP, GLAZEEBROOK $\mathrm{KN}$. Idiopathic granulomatous mastitis: imaging update and review. Insights Imaging 2016; 7: 531-539. https://doi. org/10.1007/s13244-016-0499-0

[5] BROWN KL, TANG PHL. Postlactational tumoral granulomatous mastitis: A localized immune phenomenon. Am J Surg 1979; 138: 326-329. https://doi.org/10.1016/00029610(79)90397-0

[6] ALTINOPRAK F, KARAKECE E, KIVILCIM T, DIKICIER E, CAKMAK G et al. Idiopathic granulomatous mastitis: an autoimmune disease? ScientificWorldJournal 2013; 2013: 148727. https://doi.org/10.1155/2013/148727

[7] ZHOU F, YU LX, MA ZB, YU ZG. Granulomatous lobular mastitis. Chronic Dis Transl Med 2016; 2:17-21. https://doi. org/10.1016/j.cdtm.2016.02.004

[8] YUKAWA M, WATATANI M, ISONO S, FUJIWARA Y, TSUJIE $M$ et al. Management of granulomatous mastitis: a series of 13 patients who were evaluated for treatment without corticosteroids. Int J Surg 2015; 100: 774-782. https:// doi.org/10.9738/INTSURG-D-14-00231.1

[9] ALTINOPRAK F., KIVILCIM T, OZKAN OV. Aetiology of idiopathic granulomatous mastitis. World J Clin Cases 2014; 2: 852-858. https://doi.org/10.12998/wjcc.v2.i12.852

[10] BANI-HANI KE, YAGHAN RJ, MATALKA II, SHATNAWI NJ et al. Idiopathic granulomatous mastitis: time to avoid unnecessary mastectomie. Breast J 2014; 10: 318-322. https://doi.org/10.1111/j.1075-122X.2004.21336.x

[11] KIYAK G, DUMLU EG, KILINC I, TOKAC M, AKBABA $S$ et al. Management of idiopathic granulomatous mastitis: dilemmas in diagnosis and treatment. BMC Surg 2014; 14: 66-70. https://doi.org/10.1186/1471-2482-14-66 
[12] NEEL A, HELLO M, COTTEREAU A, GRAVELEAU J, DE FAUCAL L. Long-term outcome in idiopathic granulomatous mastitis: a western multicentre study. QJM 2013; 106: 433-441. https://doi.org/10.1093/qjmed/hct040

[13] OMRANIPOUR R, MOHAMMADI SF, SAMIMI P. Idiopathic granulomatous lobular mastitis - report of 43 cases from iran; introducing a preliminary clinical practice guideline. Breast Care (Basel) 2013; 8: 439-443. https://doi. org/10.1159/000357320

[14] HOVANESSIAN LJ, PEYVANDI B, KLIPFEL N, GRANT E, IVENGAR G. Granulomatous lobular mastitis: imaging, diagnosis, and treatment. Am J Roentgenol 2009; 193: 574581. https://doi.org/10.2214/AJR.08.1528

[15] BINESH F, KARGAR S, ZAHIR ST, BEHNIAFARD N, NAVABI $\mathrm{H}$ et al. Idiopathic Granulomatous Mastitis, a Clinicopathological Review of 22 Cases. J Clin Exp Pathol 2014, 4: 157-160. https://doi.org/10.4172/2161-0681.1000157

[16] SRIPATHI S, AVACHIT A, BALA A, KADAVIGERE R, KUMAR S. Idiopathic granulomatous mastitis: a diagnostic dilemma for the breast radiologist. Insights Imaging 2016; 7: 523-529. https://doi.org/10.1007/s13244-016-0497-2

[17] GRAZIANP L, BITENCOURT AV, DA SILVA CB, GUATELLI CS, SOUZA JA et al. Imaging features of idiopathic granulomatous mastitis - Case report. Rev Assoc Med Bras 2016; 62: 303-306. https://doi.org/10.1590/1806-9282.62.04.303
[18] POYRAZ N, EMLIK GD, BATUR A, GUNDEZ E, KESKIN E et al. Magnetic Resonance Imaging Features of Idiopathic Granulomatous Mastitis: A Retrospective Analysis. Iran J Radiol 2016; 13: e20873. https://doi.org/10.5812/iranjradiol.20873

[19] GAUTIER N, LALONDE L, TRAN-THANH D, EL KHOURY M, DAVID J et al. Chronic granulomatous mastitis: Imaging, pathology and management. Eur J Radiol 2013; 82: 165-175. https://doi.org/10.1016/j.ejrad.2012.11.010

[20] GURLEIYK G, AKTEKIN A, AKER F, KARAGULLE H, SAGLAMC A. Medical and surgical treatment of idiopathic granulomatous lobular mastitis: a benign inflammatory disease mimicking invasive carcinoma. J Breast Cancer 2012; 15: 119-123. https://doi.org/10.4048/jbc.2012.15.1.119

[21] LEUNG YY, YAO HUI LL, KRAUS VB. Colchicine--Update on mechanisms of action and therapeutic uses. Semin Arthritis Rheum 2015; 45: 341-350. https://doi.org/10.1016/j. semarthrit.2015.06.013

[22] NUNEZ GUILLEN ME, DA SILVA EMIM JA, SOUCCAR C, LAPA AJ. Analgesic and anti-inflammatory activities of the aqueous extract of Plantago major L. Int J Pharmacogn 1997; 35: 99-104. https://doi.org/10.1076/phbi.35.2.99.13288 